Yamashita I (2016) Biological path for functional nanostructure fabrication and nanodevices.

Surface Innovations 4(3): 111-120,

http://dx.doi.org/10.1680/jsuin.16.00015
Surface Innovations

Volume 4 Issue SI3

Review Article

Received 19/08/2016

Published online 28/10/2016

Keywords: bioinspired/nanomaterials/nanotechnology ice

Accepted 03/10/2016

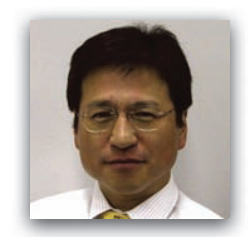

\title{
Biological path for functional nanostructure fabrication and nanodevices
}

Ichiro Yamashita $\mathrm{PhD}$ *

Professor, Graduate School of Materials Science, Nara Institute of Science and Technology, Ikoma, Japan

The fabrication of nanostructures by biomolecules is proposed. The process utilises biotemplated biomineralisation of inorganic materials and self-assembly, termed the 'bio-nano process' (BNP). Artificial proteins are designed to construct functional nanometric structures in combination with top-down miniaturisation technologies. Genetically modified cage-shaped proteins, ferritin and deoxyribonucleic acid-binding protein from starved cells, are the most studied biotemplates for the BNP. The inner cavity is used as a spatially restricted chamber for the synthesis of homogeneous metal, metal-complex and semiconductor nanoparticles (NPs). Proteins with NP cores are delivered onto specific substrate locations or carbon nanotube surfaces by electrostatic interaction or specific binding peptides. These NPs realise a variety of functions such as charge storage nodes for floating gate memory, catalysts for carbon nanotube growth, quantum wells in heterogeneous junctions and nanoetching masks. Cage-shaped protein-based bioconjugates play an important role and expand the application fields. The BNP is capable of producing functional nanostructures that are otherwise impossible through other methods.

\section{Introduction}

For the past 50 years, miniaturisation techniques have been evolving and devices are shrinking, while electronic device performance has doubled approximately every 2 years. ${ }^{1-3}$ The frontier of technology is now approaching the nanometre size, with nanometric materials/structures referred to as 'nanotechnology'. One important area in nanotechnology is biotemplated functional nanostructure fabrication. Nanofabrication technology can not only make simple nanostructures but can also produce the key components of higher-order structures, where process integration and the combination of top-down and bottom-up technology are essential. Studies on the application of biotemplates progress towards the production of more advanced devices and expand the field.

Biomolecules are the products of more than a billion years of evolution. Biomolecules are nanometre sized and can selforganise into cells, make up organs and become living beings metres in size. These features indicate that biomolecules are ideal nanoblocks for the nanofabrication of functional structures. In particular, three characteristics are fascinating. Firstly, protein molecule structures are identical down to the atomic scale and the synthesised amino acid chains fold into specific tertiary structures based on deoxyribonucleic acid (DNA) information. Secondly, biomolecules can self-assemble to form functional nanostructures. The process usually requires very little or no energy from outside. Thirdly, some proteins have surfaces on which amino acids can be arranged in such a way as to sequester inorganic materials selectively (i.e. biomineralisation). Biomineralisation bridges proteins and inorganic materials. ${ }^{4-6}$ Based on biomineralisation, biotemplated inorganic nanostructures can be produced.

Within this field, the author has proposed a biological method named the 'bio-nano process' (BNP), which combines biotemplated nanofabrication and top-down technology to produce nanofunctional structures (see Figure 1). ${ }^{7}$

The BNP has a wide range of application areas. The author has focused on research studies which utilise the cage-shaped proteins, ferritin and DNA-binding protein from starved cells (Dps). Both proteins are members of the ferritin superfamily and exist widely in many biological species. ${ }^{8-10}$ Ferritin is a ubiquitous iron (Fe) storage protein which keeps the homeostasis of iron ions. It is a 24-mer with outer and inner diameters of 12 and $7 \mathrm{~nm}$ respectively (Figure 2). Dps is a DNA-binding protein from starved cells that protects the DNA under hazardous conditions. ${ }^{11,12}$ Its outer and inner diameters are 9.5 and $4.5 \mathrm{~nm}$ respectively (Figure 2). Both proteins have narrow channels along the threefold axis connecting the cavity and the outside of the protein, through which ions can enter to produce a nanocore under certain conditions.

This paper describes several aspects of the BNP, specifically, biotemplated nanoparticle (NP) synthesis in cage-shaped proteins, delivery/arraying of the NPs by the protein shell on substrate/ nanomaterial and device applications. Bioconjugation of carbon nanotubes (CNTs) and cage-shaped proteins along with potential applications is also shown. 


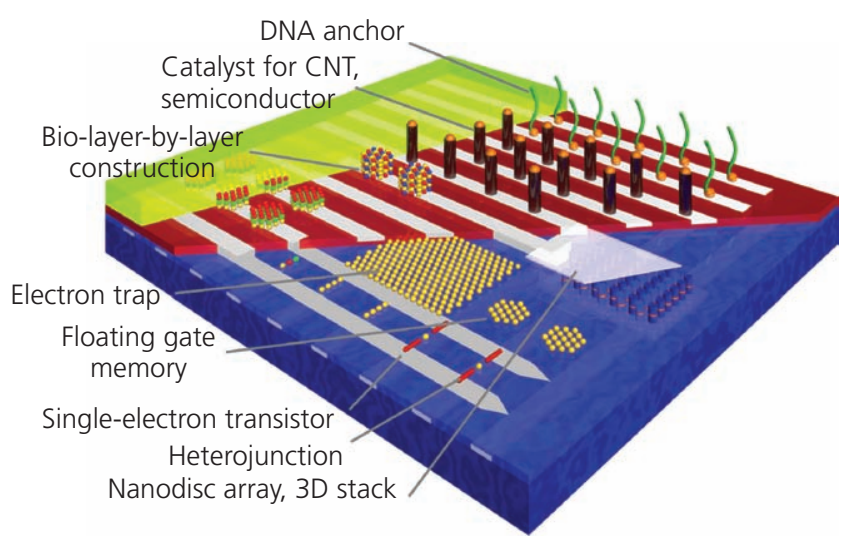

Figure 1. Schematic diagram showing examples of key components (i.e. functional nanostructures) of nanodevices produced by way of the BNP. The BNP is an integration technology combining bottom-up biotechnology and top-down technology

\section{NP synthesis in cage-shaped proteins}

NPs are fundamental elements in nanotechnology, and their production processes have been the focus of many studies. Cageshaped proteins are employed as a spatially restricted chemical chamber (i.e. a biotemplate). The inner cavity shapes are atomically identical, and in this way NPs synthesised in the cavity are homogeneous. There have been reports on metal, and metal oxide, semiconductor NP syntheses. In particular, semiconductor NPs are attracting interest. Electron energy levels depend on the NP's size and shape, and these NPs can be used as quantum dots and photofluorescence markers.

Ferritin synthesis of cadmium sulfide (CdS) NPs with diameters of 2.5 and $4.5 \mathrm{~nm}$ by incremental addition of source ions was reported by Wong and Mann. ${ }^{13}$ Yamashita et al. ${ }^{14}$ first succeeded in a onepot synthesis of compound semiconductor cadmium selenide (CdSe) NPs in the apoferritin cavity (Figure 3). The chemical reaction between cadmium (II) $\left(\mathrm{Cd}^{2+}\right)$ ions and selenium anions $\left(\mathrm{Se}^{2-}\right)$ is very fast and induces cadmium selenide aggregation quickly. Yamashita et al. ${ }^{14}$ designed an SCRY. In the SCRY,

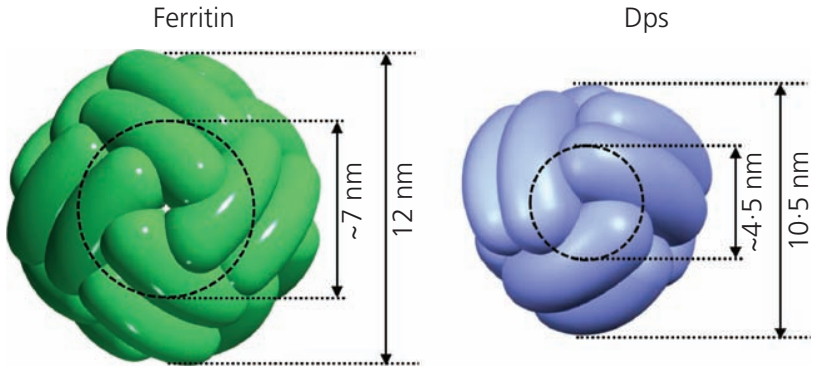

Figure 2. Schematic drawing of cage-shaped proteins, ferritin and Dps. Ferritin is composed of 24 subunits; Dps of 12 subunits

cadmium (II) ions are stabilised by excess ammonia, forming stable tetraaminecadmium ions $\left(\mathrm{Cd}\left(\mathrm{NH}_{3}\right)_{4}{ }^{2+}\right)$, and selenium cations are supplied slowly by degrading selenourea. Cadmium (II) ions are concentrated at negative collective amino acids' area on the cavity surface, and cadmium selenide nuclei are formed. Once the nuclei form, the cadmium selenide surface works as a self-catalyst and cadmium selenide semiconductor NPs are synthesised quickly.

The author optimised the SCRY reaction solution; $0.3 \mathrm{mg} / \mathrm{ml}$ ferritin, $1 \mathrm{mM}$ cadmium acetate, $1 \mathrm{mM}$ selenourea, $7.5 \mathrm{mM}$ ammonia water and $40 \mathrm{mM}$ ammonia acetate produced homogeneous cadmium selenide NPs in the cavity. ${ }^{14}$ After removal of the protein shell by $500^{\circ} \mathrm{C}$ heat treatment, a set of moderately sharp X-ray powder diffraction peaks from NPs was observed and the core structure was determined to be a cubic phase (zinc ( $\mathrm{Zn}$ ) blend) cadmium selenide with a little hexagonal phase (wurtzite). The average diameter of the obtained cadmium selenide cores was $7 \mathrm{~nm}$, which is the same as that of the apoferritin cavity. The standard deviation of the diameters was less than $10 \%$ of the average diameter.

The author applied the SCRY to zinc selenide (ZnSe). Zinc selenide NPs were synthesised in the cavities by using three recombinant apoferritins. It was proved that zinc ions accumulated at the negative glutamic acid residues on the cavity cdse

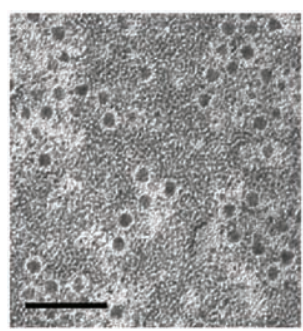

$\mathrm{ZnSe}$

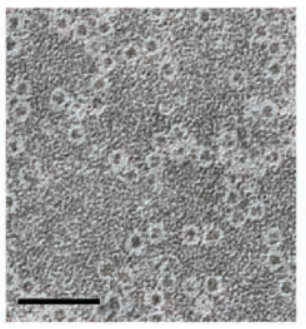

CdS

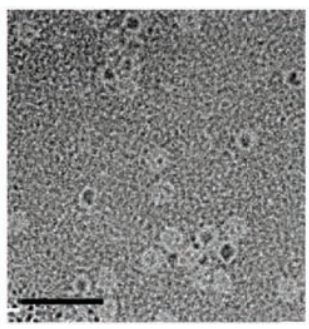

Cus

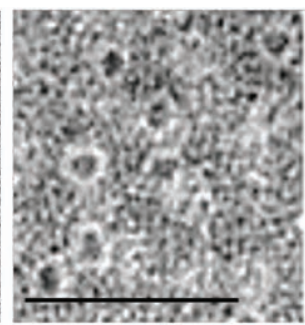

Figure 3. Transmission electron microscopy (TEM) images of some NPs artificially synthesised in ferritin cavity by a slow chemical reaction system (SCRY). The images were stained by $1 \%$ aurothioglucose, which is too large to enter and stain the cavities. The scale bars are $50 \mathrm{~nm}$ 
surface and where zinc selenide nuclei was formed. ${ }^{15}$ The author also applied the SCRY, replacing selenourea with thiourea and successfully producing cadmium sulfide, zinc sulfide $(\mathrm{ZnS})$, copper (II) sulfide (CuS), silver sulfide (AgS) and gold (I) sulfide $\left(\mathrm{Au}_{2} \mathrm{~S}\right)$ NPs (Figure 3). ${ }^{14-18}$

\section{Delivery and arraying of NPs to the designated positions}

The outer surface of cage-shaped proteins can be utilised to deliver the NPs inside the designated areas on a substrate. Precise control of electrostatic interaction between a native or mutant cage-shaped protein and an electrostatically modified substrate facilitates the successful delivery of single NPs (Figure 4). ${ }^{19,20}$ At neutral $\mathrm{pH}$, ferritin is negatively charged and the silicon surface is highly negatively charged. The author modified the silicon surface by positive 45-nm-dia. circular Aptes patterns. Ferritin was genetically modified to display 24 positively charged lysine residues on the outer surface. A numerical Poisson-Boltzmann equation calculation generated a three-dimensional (3D) electrostatic potential distribution showing a funnel-shaped path for ferritin to reach the positive nanodisc centre under appropriate ionic strength. When one ferritin molecule is adsorbed, the positive potential by Aptes was cancelled by the adsorbed ferritin and another ferritin cannot approach the Aptes disc.

Following the numeric calculation, adsorption experiments were carried out. Figure 5 shows a scanning electron microscopy (SEM) image of the obtained single NP positioning with $200-\mathrm{nm}$ intervals. ${ }^{20}$ It was confirmed that precisely controlled electrostatic interaction could place a single ferritin on an Aptes disc one by one. The delivered NPs subsequently serve as catalysts for CNTs and metal-induced lateral crystallisation or are used in other nanostructure constructions.

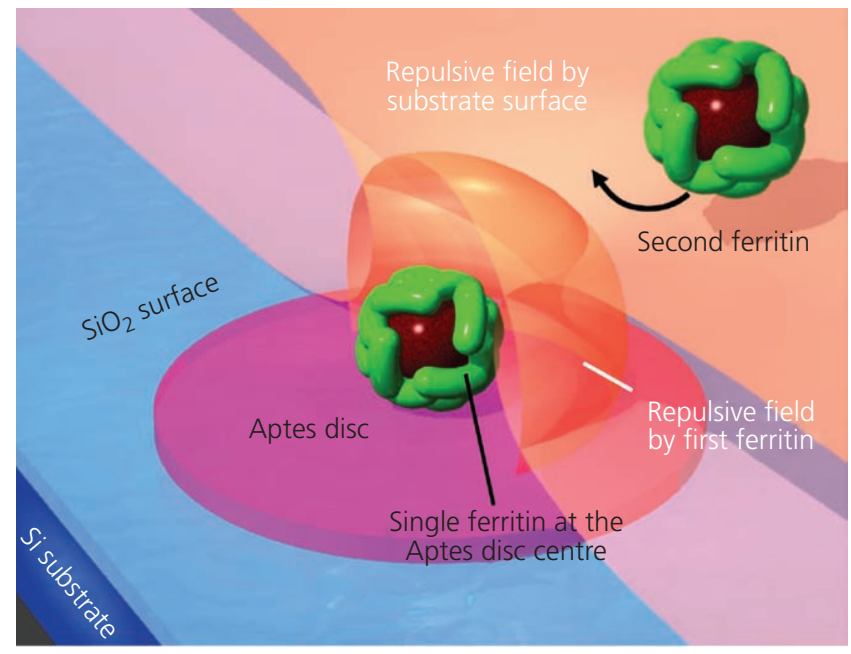

Figure 4. Schematic diagram of single ferritin adsorption onto a 3-aminopropyltriethoxysilane (Aptes) disc. The negatively charged enhanced ferritin adsorbs onto the Aptes disc centre and the second approaching ferritin will be repelled
Another approach to NP delivery is the use of target-specific binding peptides. Target-specific binding peptides can be obtained by the biopanning method, and the most used method is the phage display method. The target-specific binding peptides recognise some specific characteristic of the inorganic material surface and strongly bind to the surface. But the details of mechanism remain unclear. It is also generally shown that the peptides enhance mineralisation of the target inorganic materials from ions in aqueous solution. The nuclei of the target material may be stabilised by the peptides.

The ferritin outer surface was modified by a titanium (Ti)-binding peptide ${ }^{21,22}$ and used to deliver NPs to the titanium surface. ${ }^{23,24} \mathrm{~A}$ hexagonal $1 \cdot 5$-nm-thick titanium pattern with a diagonal length of $130 \mathrm{~nm}$ was made on a silicon substrate with a silicon dioxide $\left(\mathrm{SiO}_{2}\right)$ surface layer. A solution of the mutant ferritin with a core was put on the substrate, and after letting the solution stand for $1 \mathrm{~h}$, it was intensely washed by buffer and pure water. SEM observation clearly showed that the mutant ferritin was bound only on the titanium pattern. Moreover, fast Fourier analysis indicated that the mutant ferritins are hexagonally close packed (Figure 6). ${ }^{24}$ This delivery and arraying method promises the realisation of miniband NP structures by a simple process.

Sano et al. ${ }^{25,26}$ applied this titanium-binding recombinant ferritin to produce a $3 \mathrm{D}$ nanostructure in a layer-by-layer manner. A monolayer of recombinant ferritins with an NP core was formed on a titanium pattern in aqueous solution. On this first ferritin layer, the first layer of titania $\left(\mathrm{TiO}_{2}\right)$ was biomineralised by the recombinant ferritins. The formed titania layer then served as a binding target for a second titanium-binding recombinant ferritin. By repeating these binding and biomineralisation cycles, 3D multilayered structures were produced. Since there was no limit

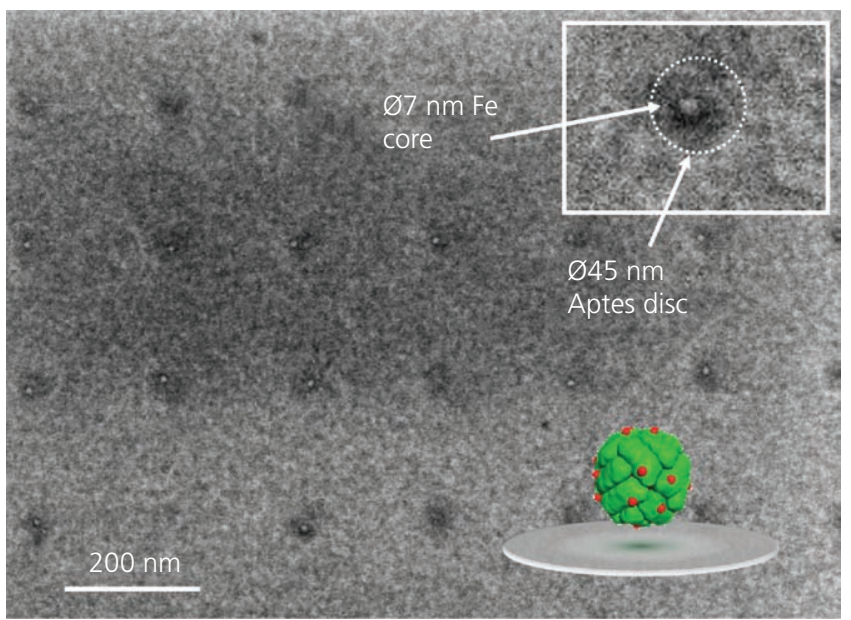

Figure 5. SEM image of a single mutant ferritin on 45-nm Aptes disc. Iron oxide NPs in ferritins are observed as bright white dots, whereas Aptes discs are observed as dark areas. Each Aptes disc has a single ferritin molecule 

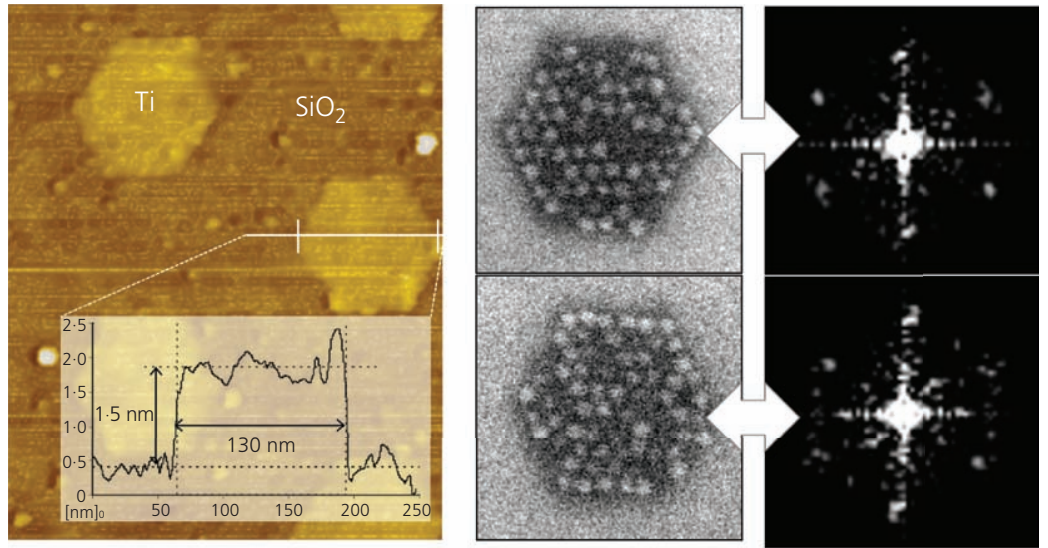

Figure 6. Atomic force microscopy (AFM) image of titanium hexagonal pattern (left); SEM image of typical mutant ferritin adsorption on the titanium pattern at pH 6.7 and 7.4 (centre); fast Fourier transform of the corresponding SEM image (right)

for the accommodated NP material, NPs in each layer were different from those in another layer. ${ }^{26}$

It is also possible that target-specific peptides endow ferritins with attractive force among them. Kase et $a l^{27}$ obtained a carbonaceous material-binding peptide by using the phage display method. The peptide was named NHBP1. Its sequence, D-Y-F-SS-P-Y-E-Q-L-F, is highly insoluble in water (i.e. hydrophobic). ${ }^{27}$ The author genetically modified ferritin the subunit and let ferritin display 24 NHBP1 peptides. The recombinant ferritin not only had the ability to bind to the carbonaceous material but also attracted other recombinant ferritins through hydrophobic interaction. The author also found that protein-protein and protein-substrate interactions were controlled by buffer solution conditions. By studying the conditions in detail, it became possible for the recombinant ferritin to fabricate a two-
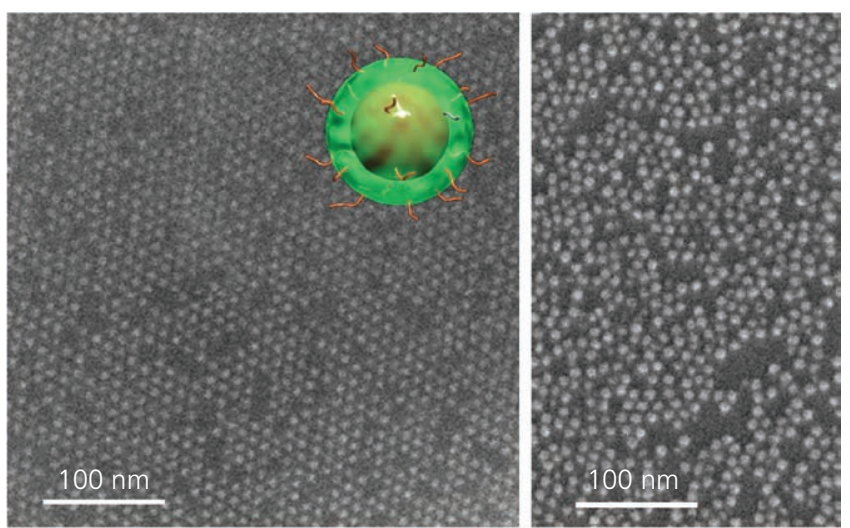

Figure 7. SEM image of hexagonally packed array of mutant ferritins with a peptide which binds specifically carbonaceous surface (left); SEM image of ferritin without target-specific peptides (right). Both ferritins were applied to the silicon surface in the same process dimensional (2D) array directly on the silicon substrate (Figure 7). ${ }^{28,29}$

Using the same recombinant carbonaceous material-binding ferritin, Ikezoe et al..$^{30}$ used the three-phase contact line (air, ferritin solution and silicon substrate) as the starting position of crystallisation and succeeded in making a much larger 2D crystal of the ferritins with an oxide core. Tsukamoto et al. ${ }^{31}$ established a simple procedure which can produce a $2 \mathrm{D}$ crystalline ferritin array by the spin-coating method. The substrate was firstly covered with poly(ethylene glycol) (Peg). The recombinant ferritins with NHBP1 peptides were spin-coated on the substrate with a thin Peg surface layer. This simple process was proved to produce a $2 \mathrm{D}$ crystalline ferritin array with a domain size of $200 \mathrm{~nm}^{31}$

\section{Applications of NPs produced and arrayed by ferritin molecules: floating gate memory}

Ferrihydrite and cobalt (II,III) oxide $\left(\mathrm{Co}_{3} \mathrm{O}_{4}\right)$ NPs synthesised in apoferritin or Dps were one or two dimensionally arrayed and used as charge storage nodes in a floating nanodot gate memory (FNGM). ${ }^{32-38}$ A metal-oxide-semiconductor field-effect transistor (Mosfet) with a tunnel silicon dioxide layer $3 \mathrm{~nm}$ thick above the channel was modified by a monolayer array of recombinant ferritin with artificially synthesised NPs. The density was around (5-8) $\times 10^{11} / \mathrm{cm}^{2}$ for ferritin and up to $1.8 \times 10^{12} / \mathrm{cm}^{2}$ for Dps. The protein shell was eliminated by heat treatment at $500^{\circ} \mathrm{C}$ for $10 \mathrm{~min}$ in oxygen gas ore ultraviolet/ozone treatment. AFM, Fourier transform infrared spectroscopy and X-ray photoelectron spectroscopy measurements confirmed the complete elimination of the protein shell. The obtained NP monolayer was embedded in a 20 -nm-thick control silicon dioxide layer. After the electrodes were deposited, post-metal annealing under forming gas $\left(10 \%\right.$ hydrogen $\left(\mathrm{H}_{2}\right)$ : $90 \%$ nitrogen $\left.\left(\mathrm{N}_{2}\right)\right)$ was carried out at 


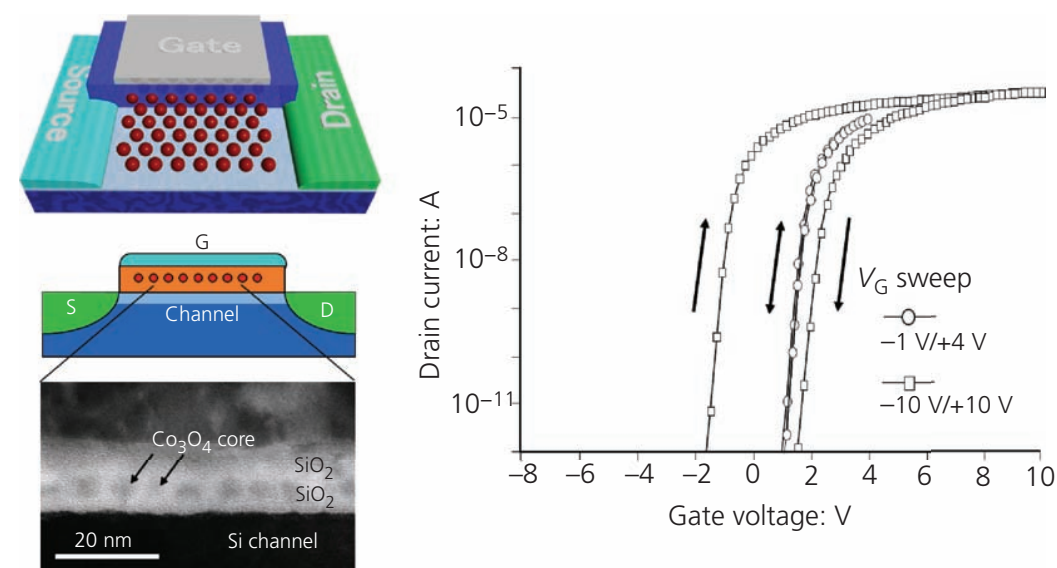

Figure 8. Schematic drawing of FNGM and the cross-sectional TEM of the device showing cobalt (Co) NPs embedded in control silicon dioxide layer (left); $I_{D}-V_{G}$ characteristics of FNGM with cobalt NPS synthesised and arrayed by ferritin (right)

$450^{\circ} \mathrm{C}$ for $1 \mathrm{~h}$. TEM observation confirmed that each NP was completely surrounded by silicon dioxide (Figure 8).

Figure 8 shows typical $I_{\mathrm{D}}-V_{\mathrm{G}}$ curves of the FNGM by the BNP. A narrow gate voltage sweep produced the typical $I_{\mathrm{D}}-V_{\mathrm{G}}$ curve of a normal n-channel Mosfet, indicating that the Mosfet functioned normally. When the gate voltage was swept from -10 to $+10 \mathrm{~V}$, the $I_{\mathrm{D}}-V_{\mathrm{G}}$ curves showed hysteresis and a threshold voltage $\left(V_{\mathrm{th}}\right)$ at which $I_{\mathrm{D}}$ shuts off, depending on the sweep direction.

When the positive gate voltage was high enough, the electrons in the n-channel tunnelled into the NP array and shut off the drain current at the higher gate voltage. In contrast, when the negative gate voltage was low enough, holes were stored in the NP array and worked in reverse. This hysteresis demonstrated that the FNGM with NPs produced and arrayed by apoferritin can work as memory.

The endurance test of the FNGM confirmed that degradation was negligible up to $10^{5}$ cycles of program/erase operation. The retention time or charge confinement time was measured up to $10000 \mathrm{~s}$, and it was confirmed that the charge can be stored for practical lengths of time. ${ }^{36}$

\section{CNTs and cage-shaped proteins}

Homogeneous NPs synthesised in apoferritin molecules are suitable catalysts for size-regulated CNT growth. Several groups have used ferritin NPs to synthesise CNTs. ${ }^{39-44} \mathrm{Kim}$ et al. ${ }^{39}$ first synthesised CNTs by using ferritin. Kramer et al. ${ }^{40}$ used Dps to produce smaller catalyst NPs for CNT synthesis. Kumagai et al. ${ }^{43}$ achieved position-controlled vertical growth of CNTs by using a periodically placed single ferritin NP array. Sugano et al. ${ }^{44}$ used a CNT forest grown from a ferritin iron oxide core array to fabricate a micromechanical resonator-type gas analyser. They selectively placed ferritins with iron oxide cores on the resonator and, after protein elimination, produced the CNT forest by using the iron oxide cores as catalysts. The density and the location of the CNT forest were well controlled. The produced analyser with the CNT forest as gas adsorber showed high sensitivity.

\section{Bioconjugates of CNTs and Dps for dye-sensitised solar cells}

Recently the author has been utilising bioconjugates of CNTs and Dps protein for the BNP. The subunit of Dps from Listeria innocua (rLiDps) was modified with the carbonaceous materialbinding peptide NHBP1 to make bioconjugates with CNTs. The NHBP1 peptide was added to the N-terminus of the LiDps subunit and expressed in Escherichia coli. It was found that the mutant proteins self-assembled into a cage-shaped protein, named NHBP-LiDps. NHBP-LiDps retained core formation ability, and NPs of iron oxide, compound semiconductors, cobalt (II,III) oxide and so on could be synthesised. After core formation, a nanostructure in which NHBP-LiDps with NP cores surrounded by CNTs was constructed by simply mixing up CNTs and NHBP-LiDps (CNT-Dps conjugates). ${ }^{45}$ Since the protein with carbonaceous material-binding peptides solubilises CNTs under mild conditions, intact $\mathrm{CNT}$ properties are preserved.

The electronic properties of the single-walled carbon nanotubes (SWCNTs) conjugated with NHBP-LiDps with a cobalt (II,III) oxide (NHBP-LiDps(cobalt (II,III) oxide) core were characterised. ${ }^{46}$ The NHBP-LiDps(cobalt (II,III) oxide) nanostructures were mixed with the SWCNTs in $20 \mathrm{mM}$ 4-(2-hydroxyethyl)-1-piperazineethanesulfonic acid)-sodium hydroxide $(\mathrm{NaOH})(\mathrm{pH} 7.5)$ in a final concentration of $0.3 \mathrm{mg} / \mathrm{ml}$ each. Ultrasonication was applied for $5 \mathrm{~min}$, and the solution was centrifuged at 15000 revolutions $/ \mathrm{min}$ for $10 \mathrm{~min}$ at $4^{\circ} \mathrm{C}$. The supernatant was observed by TEM, showing that SWCNTs were covered with NHBP-LiDps(cobalt (II,III) oxide), and the 2-nmthick protein shell provided a gap between SWCNTs and cobalt 
(II,III) oxide NPs. It was also demonstrated that the same nanostructures could be produced using ferritin molecules.

The solution of SWCNTs conjugated with NHBP-LiDps(cobalt (II, III) oxide) was dropped onto a pair of gold/chromium (Cr) electrodes with a $1-2 \cdot 5-\mu \mathrm{m}$ gap and dried. The high-density adsorption was realised by dielectrophoresis assembly with $1 \mathrm{MHz}$ $6 \mathrm{~V}$ for $5-10 \mathrm{~s}$. The sample was baked at $110^{\circ} \mathrm{C}$ for $5 \mathrm{~min}$ to remove any residual liquids. Figure 9 shows the current-voltage characteristics of the buffer-free bioconjugates network. The current suppression at low bias voltage is considered to be the effect of Coulomb blockade. It was indicated that tunnelling junctions between CNTs and some of the NPs surrounding them were formed and that the NPs worked as a quantum well. It was also suggested that the formation of conductive paths as a result of cobalt oxide reduction within NPs caused the hysteresis. These results indicate that the bioconjugates can be a new type of a heteromaterial fusing inorganic materials and proteins. Novel properties generated by the conjugation are expected.

Dps has both N- and C-termini extending outside in the solution. Inoue et al. ${ }^{47,48}$ added a carbonaceous material-binding peptide, NHBP1, at the N-terminus and a titanium-binding peptide, miniTBP1, at the C-terminus (CDT1; Figure 10). This bifunctional CDT1 was used to improve the power conversion efficiency of a dye-sensitised solar cell (DSSC). ${ }^{47,48}$ Mixing CDT1 and SWCNTs by following the same process mentioned earlier under appropriate conditions, the SWCNTs were covered with CDT1. The obtained CDT1 and SWCNT bioconjugates were used to fabricate SWCNT-titanium nanocomplexes. Since the titanium-recognising peptide, miniTBP1, can biomineralise titanium by using a soluble precursor of titanium, titanium (IV) bis(ammonium lactato)
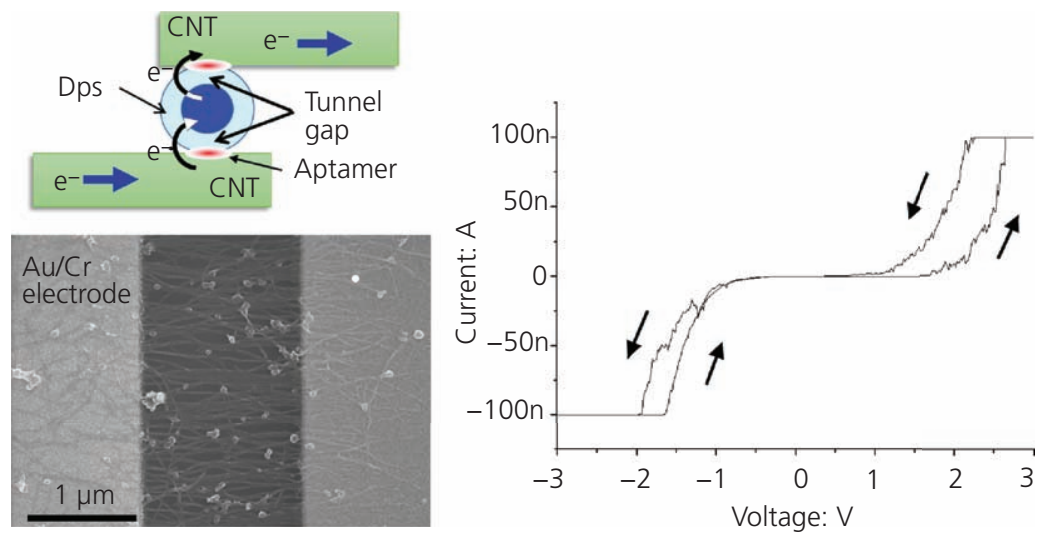

Figure 9. Schematic diagram of the tunnel junction formed in SWCNTS conjugated with NHBP-LiDps(cobalt (II,III) oxide) (top left); SEM image of the conjugate material at electrode gap (bottom left); current-voltage characteristics (right)
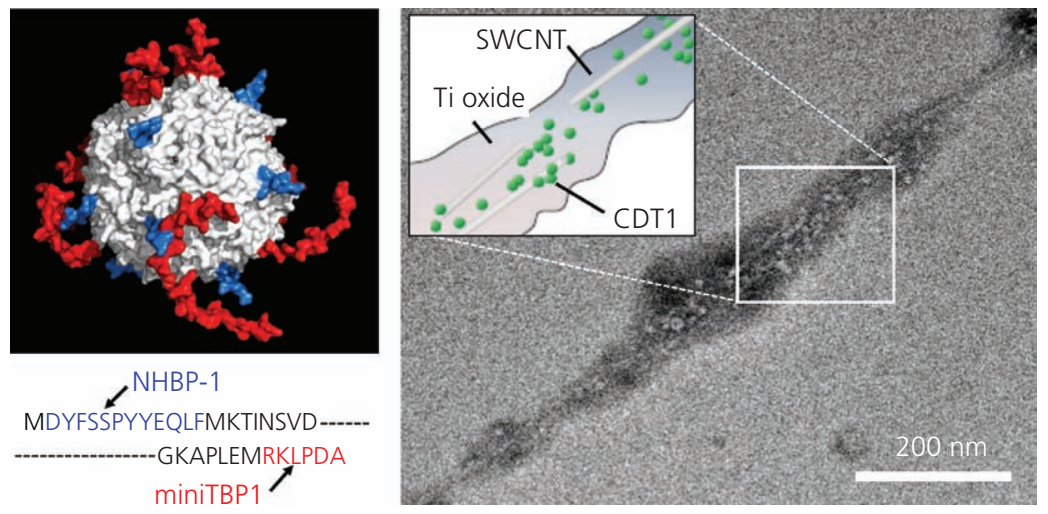

Figure 10. LiDps composed of subunits with NHBP1 at N-terminus in blue and miniTBP1 at C-terminus in red (left); SWCNT covered with CDT1 and titania layer (SWCNT/CDT1/titanium) (right) 

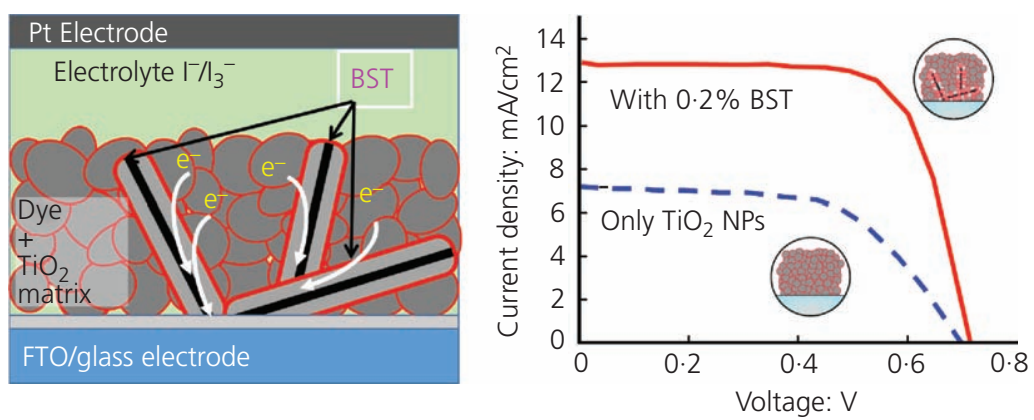

Figure 11. Schematic diagram of DSSC cross-section with BST in titania matrix layer (left); comparison of power conversion efficiency of the DSSC with titania matrix with and that of without $0 \cdot 2 \%$ BST (right)

dihydroxide (BALDH) (titanium[BALDH]), a solution of titanium [BALDH] in $50 \mathrm{mM}$ potassium phosphate buffer $(\mathrm{pH} 6.0)$ containing SWCNT/CDT1 nanocomplexes and $2 \cdot 5 \mathrm{wt} \%$ titanium [BALDH] was incubated at $24^{\circ} \mathrm{C}$ for $16 \mathrm{~h}$. After incubation, SWCNTs covered with CDT1 and a titania layer was obtained (SWCNT/CDT1/titanium: SWCNT-titanium nanocomplexes; Figure 10). Heat treatment at $450^{\circ} \mathrm{C}$ could convert the titanium layer of the nanocomplexes into the anatase phase. The obtained SWCNT-titania nanocomposite (BST) had a central highly conductive SWCNT, an electron highway surrounded by an anatase titania layer with good contact with the SWCNT. Characterisations of the BST indicated that these processes were mild and damage to the SWCNTs was minimal. ${ }^{47,48}$

The author introduced the BST into the titania matrix layer of the DSSC. In the titania matrix layer, carrier-hole recombination and trapping at grain boundaries are crucial to achieving high power conversion efficiencies. The conjugate was expected to produce an electron highway in the titania matrix, where electrons generated by the dye are transferred to the SWCNT and travel rapidly to the electrode. Figure 11 shows the power conversion efficiency of the DSSC with titania matrix, with and without $0 \cdot 2 \%$ BST. Current density improvement indicated reduction in resistance in the photovoltaic device by the nanostructure. The electrochemical impedance spectroscopy analysis showed that the charge transfer resistance at the titania/dye/electrolyte interface decreased by $33 \%$ and the transparent electrode sheet resistance by $50 \%$. These results clearly demonstrate that nanostructures produced by designed bio-supramolecules can enhance power conversion efficiency. ${ }^{48}$

\section{Bioconjugates of CNT and Dps for thermoelectric devices}

Ito et l. $^{49}$ applied CNT and Dps bioconjugates to thermoelectric devices. CNTs are a promising material expected to produce flexible thermoelectric devices based on mechanically high strength, lightness and high electric conductivity. However, there are drawbacks with CNTs such as large thermal conductivity and low Seebeck coefficient. Ito et al ${ }^{49}$ proposed a thermoelectric material composed of CNT-Dps bioconjugates, which may overcome the aforementioned drawbacks. CNTs were partially covered with NHBP-LiDps with a semiconductor core, and the mixing conditions were selected. The obtained conjugates were condensed to make a black material with several CNT/ NHBP-LiDps/CNT junctions (Figure 12). The junctions create a new type of Seebeck effect and reduce the thermal conductivity dramatically. At the junction, phonons are scattered at the CNT/ soft shell interface, which suppresses the heat flow of the conjugates' bulk material. This leads to the generation of a steep

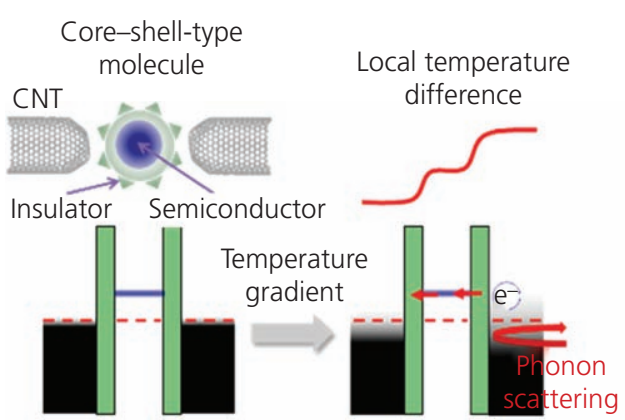

(a)

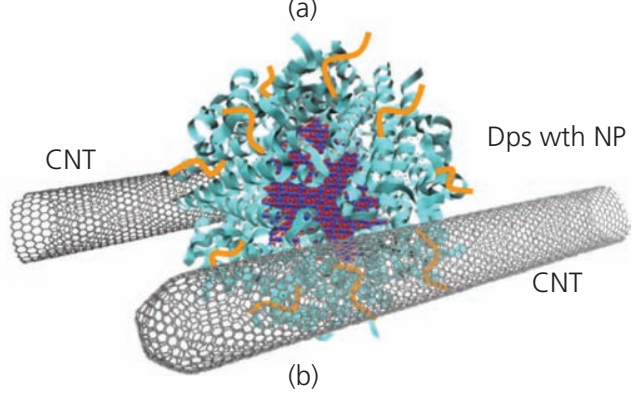

Figure 12. Schematic diagrams of (a) Seebeck effect at a biomolecular junction, (b) drawing of two Campylobacter jejuni Dps molecules bridging two CNTs. Note that the CNTs are enlarged for ease. Copyright (2014) The Japan Society of Applied Physics 
temperature gradient across the junction, and this steep temperature gradient generates an asymmetric Fermi-Dirac distribution of electrons/holes between two CNTs. This asymmetry causes electrons/holes to flow unidirectionally through the conduction or valence band of the core. ${ }^{49}$

Ito et al. ${ }^{49}$ made thin conjugate films employing NHBP-LiDps with iron oxide cores and measured the thermal and electrical properties. The results indicated the thermal conductivity was markedly suppressed, the electrical conductivity increased and the Seebeck coefficient increased. As a result, the figure of merit, $Z T=\alpha^{2} \sigma T / \kappa$ $(\alpha, \sigma, \kappa$ and $T$ are the Seebeck coefficient, electrical conductivity, thermal conductivity and absolute temperature respectively), is increased over 1000-fold. They also made the conjugates with NHBP-LiDps with cadmium selenide core, and $Z T$ was shown to increase around 66-fold (see Table 1). ${ }^{47}$ Thus, the BNP can produce nanostructures very easily under eco-friendly conditions.

\section{Conclusion and perspective}

The top-down technology of miniaturisation is powerful but cannot produce sub-10-nm structures essential for next-generation nanoelectronic devices and quantum effect devices. The BNP can produce sub-10-nm structures by combining biotemplated biomineralisation of inorganic materials and self-assembly. As demonstrated in this paper, artificial proteins are efficient at producing such nanometric structure constructions (i.e. bottom-up technology). The integration of top-down and bottom-up technologies is a promising technology for realising and producing nanoelectronic devices commercially. However, several hurdles remain until the BNP will be used in mass productions. Those hurdles include economical highly pure protein production, precise control of protein adsorption to designated surfaces and easy design and production of biotemplates or protein supramolecules with target shapes. But based on the fact that intensive basic research studies have rapidly increased the number of materials that can be synthesised in cage-shaped proteins, these limits shall soon be lifted. This biological approach will open up a new path towards the fabrication of functional nanostructures realising new devices operating on completely new mechanisms.

\section{Acknowledgements}

This paper includes works done by the researchers at Tohoku University, Tokyo Institute of Technology, Toyota Technological Institute, Nara Institute of Science and Technology, the Core Research for Evolutionary Science and Technology team of the Japan Science and Technology Agency, Panasonic Corporation and Ajinomoto Co., Inc. The author thanks L. McDowell for English proofreading.

\section{REFERENCES}

1. Moore GE (1965) Cramming more components onto integrated circuit. Electronics 38(8): 114-117.

2. Moore GE (1975) Progress in digital and electronics. Technical Digest 1975: International Electron Devices Meeting IEEE, Washington, DC, USA, pp. 11-13.

3. Gelsinger P (2006) Moore's law - the Genius lives on. IEEE SSCS Newsletter 20(3): 18-20.

4. Simkiss K and Wilbur KM (1989) Biomineralization. Academic, San Diego, CA, USA.

5. Mann S (2001) Biomineralization: Principles and Concepts in Bioinorganic Materials Chemistry. Oxford University Press, Oxford, UK.

6. Bauerlein E (2004) Biomineralization. Wiley-VCH, Cambridge, UK.

7. Yamashita I (2000) Fabrication of a two-dimensional array of nano-particles using ferritin molecule. Thin Solid Films 393(1-2): 12-18.

8. Harrison PM, Andrews SC, Artymuik PJ et al. (1991) Probing structure-function relations in ferritin and bacterioferritin. Advances in Inorganic Chemistry 36: 449-486.

9. Harrison PM and Arosio P (1996) The ferritins: molecular properties, iron storage function and cellular regulation. Biochimica et Biophysica Acta 1275(3): 161-203.

10. Massover WH (1993) Ultrastructure of ferritin and apoferritin: a review. Micron 24(4): 389-437.

11. Almirón M, Link AJ, Furlong D and Kolter R (1992) A novel DNA-binding protein with regulatory and protective roles in starved Escherichia coli. Genes \& Development 6(12B): 2646-2654.

\begin{tabular}{|c|c|c|c|c|}
\hline & $\begin{array}{l}\text { Thermal conductivity: }^{\text {a }} \\
\text { m/(W K) }\end{array}$ & $\begin{array}{c}\text { Seebeck coefficient: }^{\text {b }} \\
\mu \mathrm{V} / \mathrm{K}\end{array}$ & $\begin{array}{l}\text { Electrical conductivity: } \\
\qquad \mathrm{S} / \mathrm{cm}\end{array}$ & $\begin{array}{c}\text { Relative ZT } \\
\text { (at } 300 \mathrm{~K} \text { ) }\end{array}$ \\
\hline CNT (pristine) & $17 \pm 0 \cdot 5$ & 33 & 0.30 & 1 \\
\hline CNT/Dps(iron) & $0.13 \pm 0.03$ & $56 \pm 12$ & $0.84 \pm 0.07$ & 1071 \\
\hline $\begin{array}{l}\text { CNT/Dps(cadmium } \\
\text { selenide) }\end{array}$ & - & $28 \pm 5$ & $0.21 \pm 0.11$ & $66^{c}$ \\
\hline
\end{tabular}

\footnotetext{
${ }^{\text {a }}$ Measured in the out-of-plane direction

${ }^{\mathrm{b}}$ Measured in the in-plane direction

c Thermal conductivity of CNT/Campylobacter jejuni Dps(Fe) was used for the calculation
} 
12. Bozzi M, Mignogna G, Stefanini S et al. (1997) A novel nonheme iron-binding ferritin related to the DNA-binding proteins of the Dps family in Listeria innocua. Journal of Biological Chemistry 272(6): 3259-3265.

13. Wong KKW and Mann S (1996) Biomimetic synthesis of cadmium sulphide-ferritin nanocomposites. Advanced Materials 8(11): 928-932.

14. Yamashita I, Hayashi J and Hara M (2004) Bio-template synthesis of uniform CdSe nanoparticles using cage-shaped protein, apoferritin. Chemistry Letters 33(9): 1158-1159.

15. Iwahori K, Yoshizawa K, Muraoka M and Yamashita I (2005) Fabrication of $\mathrm{ZnSe}$ nanoparticles in the apoferritin cavity by designing a slow chemical reaction system. Inorganic Chemistry 44(18): 6393-6400.

16. Iwahori K and Yamashita I (2008) Size-controlled one-pot synthesis of fluorescent cadmium sulfide semiconductor nanoparticles in an apoferritin cavity. Nanotechnology 19(49): 495601.

17. Iwahori K, Takagi R, Kishimoto N and Yamashita I (2011) A size controlled synthesis of $\mathrm{CuS}$ nano-particles in the protein cage, apoferritin. Materials Letters 65(21-22): 3245-3247.

18. Yoshizawa K, Iwahori K, Sugimoto K and Yamashita I (2006) Fabrication of gold sulfide nanoparticles using the protein cage of apoferritin. Chemistry Letters 35(10): 1192-1193.

19. Kumagai S, Yoshii S, Yamada K et al. (2006) Electrostatic placement of single ferritin molecules. Applied Physics Letters 88(15): 153103.

20. Yoshii S, Kumagai S, Nishio K, Kadotani A and Yamashita I (2009) Electrostatic self-aligned placement of single nanodots by protein supramolecules. Applied Physics Letters 95(13): 133702.

21. Sano K, Sasaki H and Shiba K (2005) Specificity and biomineralization activities of Ti-binding peptide-1 (TBP-1). Langmuir 21(7): 3090-3095.

22. Sano K, Ajima K, Iwahori K et al. (2005) Endowing a ferritinlike cage protein with high affinity and selectivity for certain inorganic materials. Small 1(8-9): 826-832.

23. Yamashita I, Kirimura H, Okuda M et al. (2006) Selective nanoscale positioning of ferritin and nanoparticles by means of target-specific peptides. Small 2(10): 1148-1152.

24. Matsukawa N, Nishio K, Sano K, Shiba K and Yamashita I (2009) Hexagonal close-packed array formed by selective adsorption onto hexagonal patterns. Langmuir 25(6): 3327-3330.

25. Sano KI, Sasaki H and Shiba K (2006) Utilization of the pleiotropy of a peptidic aptamer to fabricate heterogeneous nanodot-containing multilayer nanostructures. Journal of the American Chemical Society 128(5): 1717-1722.

26. Sano KI, Yoshii S, Yamashita I and Shiba K (2007) In aqua structuralization of a three-dimensional configuration using biomolecules. Nano Letters 7(10): 3200-3202.

27. Kase D, Kulp JL, Yudasaka M et al. (2004) Affinity selection of peptide phage libraries against single-wall carbon nanohorns identifies a peptide aptamer with conformational variability. Langmuir 20(20): 8939-8941.
28. Matsui T, Matsukawa N, Iwahori K et al. (2007) Realizing a two-dimensional ordered array of ferritin molecules directly on a solid surface utilizing carbonaceous material affinity. Langmuir 23(4): 1615-1618.

29. Matsui T, Matsukawa N, Iwahori K et al. (2007) Direct production of a two-dimensional ordered array of ferritinnanoparticles on a silicon substrate. Japanese Journal of Applied Physics 46(25-28): L713-L715.

30. Ikezoe Y, Kumashiro Y, Tamada K et al. (2008) Growth of giant two-dimensional crystal of protein molecules from a three-phase contact line. Langmuir 24(22): 12836-12841.

31. Tsukamoto $R$, Igarashi $M$, Samukawa $S$ and Yamashita I (2012) Fast two-dimensional ferritin crystal formation realized by mutant ferritin and poly(ethylene glycol) modified $\mathrm{SiO}_{2}$ substrate. Applied Physics Express 5(6): 065201.

32. Miura A, Hikono T, Matsumura T et al. (2006) Floating nanodot gate memory devices based in biomineralized inorganic nanodot array as a storage node. Japanese Journal of Applied Physics 45(1-3): L1-L3.

33. Yoshii S, Yamada K, Matsukawa N and Yamashita I (2005) Making monolayer of inorganic nanoparticles on silicon substrate. Japanese Journal of Applied Physics 44(3): 1518-1523.

34. Yamada K, Yoshii S, Kumagai S et al. (2007) Effects of dot density and dot size on charge injection characteristics in nanodot array produced by protein supramolecules. Japanese Journal of Applied Physics 46(11): 7549-7553.

35. Miura A, Tsukamoto R, Yoshii S et al. (2008) Non-volatile flash memory with discrete bionanodot floating gate assembled by protein template. Nanotechnology 19(25): 255201.

36. Miura A, Tanaka R, Uraoka Y et al. (2009) The characterization of a single discrete bionanodot for memory device applications. Nanotechnology 20(12): 125702.

37. Kamitake H, Uenuma M, Okamoto $\mathrm{N}$ et al. (2015) Floating gate memory with charge storage dots array formed by Dps protein modified with site-specific binding peptides. Nanotechnology 26(19): 195201.

38. Ban T, Uenuma M, Migita S et al. (2015) Ultra-short channel junctionless transistor with a one-dimensional nanodot array floating gate. Applied Physics Letters 106: 253104.

39. Kim Y, Li W, Zhang Y et al. (2001) Growth of single-walled carbon nanotubes from discrete catalytic nanoparticles of various sizes. Journal of Physical Chemistry B 105(46): 11424-11431.

40. Kramer RM, Sowards LA, Pender MJ, Stone MO and Naik RR (2005) Constrained iron catalysts for single-walled carbon nanotube growth. Langmuir 21(18): 8466-8470.

41. Jeong GH, Yamazaki A, Suzuki S et al. (2005) Cobalt-filled apoferritin for suspended single-walled carbon nanotube growth with narrow diameter distribution. Journal of the American Chemical Society 127(23): 8238-8239.

42. Takagi D, Yamazaki A, Otsuka Y et al. (2007) Gold-filled apo-ferritin for investigation of single-walled carbon nanotube growth on substrate. Chemical Physics Letters 445(4-6): 213-216. 
43. Kumagai S, Ono T, Yoshii S et al. (2010) Position-controlled vertical growths of individual carbon nanotubes using a cageshaped protein. Applied Physics Express 3(1): 015101.

44. Sugano K, Matsumoto R, Tsutsui R et al. (2016) Fabrication and characterization of a CNT forest integrated micromechanical resonator for a rarefied gas analyzer in a medium vacuum atmosphere. Journal of Micromechanics and Microengineering 26(7): 075010 .

45. Kobayashi M, Kumagai S, Zheng B et al. (2011) A watersoluble carbon nanotube network conjugated by nanoparticles with defined nanometre gaps. Chemical Communications 47(12): 3475-3477.

46. Kumagai S, Okamoto N, Kobayashi M and Yamashita I (2012) Characterisation of a carbon nanotube random network conjugated by semiconductor nanoparticles with defined nanometre-scaled gaps. Micro \& Nano Letters 7(8): 753-756.

47. Inoue I, Zheng B, Watanabe K et al. (2011) A novel bifunctional protein supramolecule for construction of carbon nanotube-titanium hybrid material. Chemical Communications 47(47): 12649-12651.

48. Inoue I, Watanabe K, Yamauchi H et al. (2014) Biological construction of single-walled carbon nanotube electron transfer pathways in dye-sensitized solar cells. ChemSusChem 7(10): 2805-2810.

49. Ito M, Okamoto N, Abe R et al. (2014) Enhancement of thermoelectric properties of carbon nanotube composites by inserting biomolecules at nanotube junctions. Applied Physics Express 7(6): 065102.

\section{HOW CAN YOU CONTRIBUTE?}

To discuss this paper, please submit up to 500 words to the journal office at journal@ice.org.uk. Your contribution will be forwarded to the author(s) for a reply and, if considered appropriate by the editor-in-chief, it will be published as a discussion in a future issue of the journal.

ICE Science journals rely entirely on contributions from the field of materials science and engineering. Information about how to submit your paper online is available at www.icevirtuallibrary.com/page/authors, where you will also find detailed author guidelines. 